\title{
Improved Memetic Algorithm for Multi-depot Multi-objective Capacitated Arc Routing Problem
}

\author{
Jie Wan ${ }^{1}$, Xinghan Chen ${ }^{1, a}$ and Ruichang $\mathrm{Li}^{1}$ \\ Hebei University of Technology, School of Economics and Management, 300401 Tianjin, China
}

\begin{abstract}
The capacitated arc routing problem (CARP) is a challenging vehicle routing problem with numerous real-world applications. In this paper, an extended version of CARP, the multi-depot multi-objective capacitated arc routing problem (MDMOCARP) is proposed to tackle practical requirements. Firstly, the critical edge decision mechanism and the critical edge random allocation mechanism are proposed to optimize edges between depots. Secondly, a novel adaptive probability of local search with fitness is proposed to improve the Decomposition-Based Memetic Algorithm for Multi-Objective CARP (D-MAENS). Compared with the D-MAENS algorithm, experimental results on MD-CARP instances show that the improved memetic algorithm (IMA) has performed significantly better than D-MAENS on convergence and diversity in the metric IGD and the metric HV.
\end{abstract}

\section{Introduction}

Capacitated Arc Routing Problem (CARP) [1] has wide real-world applications such as snow removal [2], urban waste collection [3-4], pipeline repair [5] etc. Today, these practical issues that are closely related to the logistics transportation have become very important to the government administration. For example, in order to prevent roads from freezing and reduce the traffic accidents, the UK spends millions of pounds on salting in the relevant streets each year.

With the development of society, the typical CARP model cannot meet the actual needs of complexity. Therefore, the application of the CARP model has been widely concerned by researchers. To fulfil the needs of application, some extended versions of CARP were considered, such as the multi-objective CARP [6], the multi-depot CARP [7], the periodic CARP [8] and so on.

Recently, a number of researchers have done a lot of work on multi-depot and multi-objective. Multi-depot CARP (MDCARP) is the CARP with more than one depot or one center. Amberg et al. [9] described a minimal Euler graph, and transformed the problem into the capacitated minimum spanning tree to solve MDCARP early. However, the method only applied to the undirected graph and found the local optimal solution. Kansou et al. [10] insert the ant colony into the sequence of tasks and uses heuristic information to insert the appropriate location, thus proposed a new ant colony algorithm (ACs) to solve MDCARP. Xing et al. [11] improved augment-merge, path-scanning, and the Ulusoy-split, which seeks good quality feasible initial solutions. Krushinsky et al. [7] proposed a dual-index MILP model based on asymmetric multi-depot problem. Oliveira et al. [12] proposed a problem decomposition approach. The task or customer is allocated to the depot based on the distance so that each sub-problem becomes a single depot problem. But the repair operator needs to remove duplicate tasks which lead to the new cost.

On the other hand, in the multi-objective CARP (MOCARP), Lacomme et al. [13] studied the two-objective capacitated arc routing problem model to meet the needs of Troyes in waste collection, and solved the problem by using NSGAII algorithm. Mei et al. [14] proposed the decomposition based memetic algorithm for multi-objective capacitated arc routing problem (D-MAENS), which combines the MAENS algorithm with a multi-objective evolutionary algorithm framework based on problem decomposition. Shang et al. [15] improved the update mechanism and intended to an improved decomposition-based memetic algorithm for MOCARP. Shang et al. [16] used the routing distance grouping strategy to reduce the scale of the problem in large-scale MOCARP.

However, little attention has been paid to the multi-depot multi-objective capacitated arc routing problem (MDMOCARP). In part, these observations motivated our study. Therefore, this paper contributes to constructing a MDMOCARP model. An improved memetic algorithm (IMA) with the critical edge decision

a Corresponding author: chenxh2017001@163.com 
mechanism and the critical edge random allocation mechanism is proposed. In order to control local search frequency, we develop an adaptive probability of local search. In order to better solve the MDMOCARP model and increase its application range.

This paper is organized as follows. The next section gives the mathematical description of the problem and the improved memetic algorithm. The third section presents the results of our experimental studies, which compare the D-MAENS algorithms using the MDCARP instances. The conclusion is provided in section 4 .

\section{Model establishment and algorithm improvement}

\subsection{The MDMOCARP model}

In this section, the mathematical model is described for the multi-depot multi-objective CARP. The main purpose of the model is to minimize the total cost of all the tours and to minimize the largest consumption of $m$ vehicles. Because in the actual CARP, if the area of the vehicle requiring service is expanded, it is necessary to use multiple-depot for service. At the same time, only minimizing the total cost cannot meet the actual demand. In addition, minimizing the maximum loop cost is needed to ensure the optimization effect of arc path. For convenience, the following necessary assumptions are given:

1. Each route must start and end the same depot.

2. The vehicle only serves once for the task side.

3. The total demand of served tasks cannot exceed the capacity $Q$ of the vehicle.

4. All edges in the connected graph are allowed to traversed by vehicles of different depots, but only serviced by the vehicle of the belonging depot.

Table 1. The definition of variables.

\begin{tabular}{|l|l|}
\hline Variables & Definition \\
\hline$v_{i}$ & The vertices of the graph \\
\hline$t c\left(v_{i}, v_{j}\right)$ & The cost of traversing the edge \\
\hline$s c\left(v_{i}, v_{j}\right)$ & The cost of serving the edge \\
\hline$d\left(v_{i}, v_{j}\right)$ & The service demand of the edge $\left(v_{i}, v_{j}\right)$ \\
\hline$x_{h\left(v_{i}, v_{j}\right)}^{d}$ & $\begin{array}{l}\text { The } 0 \text {-1 variable, } x_{h\left(v_{i}, v_{j}\right)}^{d}=1 \quad \text { is the depot } \\
\text { to } v_{j} ; \text { otherwise } x_{h\left(v_{i}, v_{j}\right)}^{d}=0\end{array}$ \\
\hline$y_{h\left(v_{i}, v_{j}\right)}^{d}$ & $\begin{array}{l}\text { The } 0-1 \quad \text { variable, } y_{h\left(v_{i}, v_{j}\right)}=1 \\
\text { depot } d \quad \text { and the vehicle } \quad h \quad \text { traverses } \\
\text { from } v_{i} \text { to } v_{j} ; \text { otherwise } y_{h\left(v_{i}, v_{j}\right)}^{d}=0\end{array}$ \\
\hline$d$ & The depot ID number \\
\hline$h$ & The vehicle ID number \\
\hline$n$ & The depot total number \\
\hline$m$ & The vehicle total number \\
\hline$\left|L_{h}^{d}\right|$ & $\begin{array}{l}\text { The length of the task sequence of the } \\
\text { vehicle } h \text { in the depot } d\end{array}$ \\
\hline
\end{tabular}

\begin{tabular}{|l|l|}
\hline $\operatorname{Tc}\left(\left|L_{h}^{d}\right|\right)$ & $\begin{array}{l}\text { The total cost of the } h \text { vehicle in the } \\
\operatorname{depot} d\end{array}$ \\
\hline
\end{tabular}

As Table 1 shows, the key variables are defined. The graph is presented by $G=(V, E)$, as shown in Figure 1 . $V=\left\{v_{1}, v_{2}, \cdots v_{(w+n)}\right\}$ is the set of vertices in a graph. $V_{1}=\left\{v_{w+1}, v_{w+2}, \cdots v_{(w+n)}\right\} \subseteq V$ indicates that there are $n$ depots in the graph. An edge $\left(v_{i}, v_{j}\right) \in E$ is directed edge from $v_{i}$ to $v_{j}$.

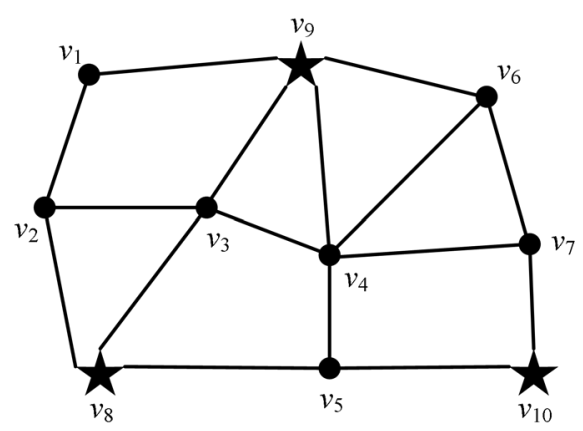

Figure 1. The schematic diagram of MDMOCARP model.

Based on that, the mathematical model can be given as follows.

$$
\begin{gathered}
\min f_{1}(x)=\sum_{d=1}^{n} \sum_{h=1}^{m} T c\left(L_{h}^{d}\right) \\
\min f_{2}(x)=\max _{1 \leq d \leq n}\left(\max _{1 \leq h \leq m}\left(T c\left(L_{h}^{d}\right)\right)\right)
\end{gathered}
$$

s.t.

$$
\begin{gathered}
T c\left(L_{h}^{d}\right)=\sum_{\left(v_{i}, v_{j}\right) \in E}\left(s c\left(v_{i}, v_{j}\right) x_{h\left(v_{i}, v_{j}\right)}^{d}+t c\left(v_{i}, v_{j}\right) y_{h\left(v_{i}, v_{j}\right)}^{d}\right) \\
x_{h\left(v_{i}, v_{j}\right)}^{d}+y_{h\left(v_{i}, v_{j}\right)}^{d}=x_{h\left(v_{k}, v_{i}\right)}^{d}+y_{h\left(v_{k}, v_{i}\right)}^{d} \\
1 \leq h \leq m, 1 \leq d \leq n \\
\sum_{d=1}^{n} \sum_{h=1}^{m} x_{h\left(v_{i}, v_{j}\right)}^{d}=1, \forall\left(v_{i}, v_{j}\right) \in E \\
\sum_{d=1}^{n} \sum_{h=1}^{m} y_{h\left(v_{i}, v_{j}\right)}^{d} \geq 1, \forall\left(v_{i}, v_{j}\right) \in E \\
\sum_{\left(v_{i}, v_{j}\right) \in E} d\left(v_{i}, v_{j}\right) x_{h\left(v_{i}, v_{j}\right)}^{d} \leq Q, \forall\left(v_{i}, v_{j}\right) \in E \\
x_{h\left(v_{i}, v_{j}\right)}^{d} \leq y_{h\left(v_{i}, v_{j}\right)}^{d}, 1 \leq h \leq m, 1 \leq d \leq n, \forall\left(v_{i}, v_{j}\right) \in E
\end{gathered}
$$

Here, the target $f_{1}$ is to minimize the total cost and the target $f_{2}$ is to minimize the largest consumption of $m$ vehicles. The constraint (3) is the total cost of the vehicle $h$ of the depot $d$. The constraint (4) ensures that vehicle $h$ start at the depot and return to it when the service is completed. The constraint (5) means that the task side is served only once. The constraint (6) is that all sides in the graph allow the vehicle to pass through indefinitely. The constraint (7) requires that the total demand cannot exceed the capacity $Q$ of the service vehicle. When the vehicle is servicing a task edge, it must pass through the task edge by constraint (8). 


\subsection{Improved memetic algorithm}

For MDMOCARP, an improved memetic algorithm (IMA) is proposed based on D-MAENS [14]. In the section, we first described the critical edge decision mechanism considering two indicators of distance from the task side to depots and demand on the task side. Then we design the critical edge's random allocation mechanism. Finally, we present the adaptive probability of local search.

\subsubsection{Critical edge decision mechanism}

In the multi-depot problem, we generally need to convert multiple depots into a single depot. Different conversion strategies affect the final optimization results. In order to gain the global optimization, this paper uses the critical edge optimize. The distance between the task edge and the depots and the demand of the task edge are the criterion for determining the critical edge.

$$
\theta<\frac{\Delta \operatorname{task}\left(v_{w 1},\left(v_{i}, v_{j}\right)\right)}{\Delta \operatorname{task}\left(v_{w 2},\left(v_{i}, v_{j}\right)\right)}+\rho^{*}\left(d\left(v_{i}, v_{j}\right) / \text { capacity }\right)
$$

Where, $\theta$ is the threshold. $\Delta \operatorname{task}\left(v_{w 1},\left(v_{i}, v_{j}\right)\right)$ and $\Delta \operatorname{task}\left(v_{w 2},\left(v_{i}, v_{j}\right)\right)$ are the distance from the edge to depot $w_{1}$ and $w_{2} \cdot d\left(v_{i}, v_{j}\right) /$ capacity denotes the proportion of the demand of the edge and the capacity of vehicle. $\rho$ refers to the weight parameter.

\subsubsection{Critical edge random allocation mechanism}

In order to efficiently handle MDMOCARP, we propose the critical edge random allocation mechanism. The critical edge random allocation mechanism is described as follows:

Algorithm 1: Critical edge random allocation mechanism
Input:
Critical edge set $T_{\left(v_{i}, v_{j}\right)} \in E$
The present best solution: $S$
Output:
The best solution: $S^{*}$
1. Randomly select $n$ edges from the set $T_{\left(v_{i}, v_{j}\right)}$
2. Assign $n$ edges randomly to a depot
3. Use the IMA algorithm to solve the problems of each
depot, and generate a global new solution
4. Replace if the offspring solution is better than the
previous solution
5 . Count the number of new solutions without
replacement with numb
6 . If numb reaches $M A X_{-}$numb, it will exit or it will be
converted to step 1

\subsubsection{Adaptive probability of local search}

In the process of evolution, local search occupied a lot of computer resources, and it is not efficient that the same local search probability is applied to the search individuals. we design an adaptive probability of local search with the fitness of the individual for multi-objective optimization. The main ideas are local search is only allowed when needed and adaptive probability which increases with the fitness of the individual.

$$
\begin{aligned}
& \varphi=\frac{f i t_{\text {ave }}-f i t_{\text {best }}}{f i t_{\text {best }}} * 100 \% \\
& S=\frac{1}{1+k * \sqrt{\frac{\sum_{i=1}^{\text {popsize }}\left(f i t_{i}-f i t_{\text {ave }}\right)^{2}}{\text { popsize }}}} \\
& p l s=\sin \left(\frac{\Pi}{2} * \frac{f i t_{\text {best }}}{\text { fit }_{i}} * S\right) \\
& P=\left\{\begin{array}{c}
p l s, \varphi>\gamma \\
0, \varphi \leq \gamma
\end{array}\right.
\end{aligned}
$$

Here, fit $t_{\text {ave }}$ and fit $_{\text {best }}$ represent the average value of fitness of the individual and the best value of fitness. the deviation rate denoted by $\varphi$ in the formula (10). fit $_{i}$ is the fitness of the $i . k$ means adjustment factor, $k=0.1$. popsize is the population size. $P$ represents the adaptive probability of local search. $\gamma$ is set as 0.01 .

\section{Experiment analysis}

\subsection{MDCARP instances and parameter settings}

The MDCARP instances [10] called md-Golden are obtained by adding depots in the basic CARP instances. It includes the 23 CARP instances. The size of instances is from 7 to 27 vertices and 11-55 required edges. It has two depots, at the first node and at the final node.

The IMA was implemented in Visual C language, and all experiments were run on a personal computer with the 3.0GHZ processor and $8 \mathrm{~GB}$ memory. The parameters were: The population size is 30 . The maximum number of iterations was 1000 . The maximum number MAX_numb of depot cycle was 100. The parameter settings of the probability of local search for D-MAENS was 0.2 .

\subsection{IMA algorithm threshold value $\theta$ analysis}

The IMA algorithm uses threshold $\theta$ to determine critical edge, so we need analyze it. Seven kinds of thresholds $\theta$ were analyzed, which were $0.5,0.6,0.7$, $0.8,0.9,1.0,1.1$, respectively with the instance g12. IMA algorithm was run 10 times independently, and gained the average of the total cost. The results are presented in Figure 2.

In the Figure 2, it can be found that when the thresholds are in the middle of 0.6 and 0.8 , the total cost can get better optimization results. Therefore, the threshold of 0.7 is more reasonable in the IMA. 


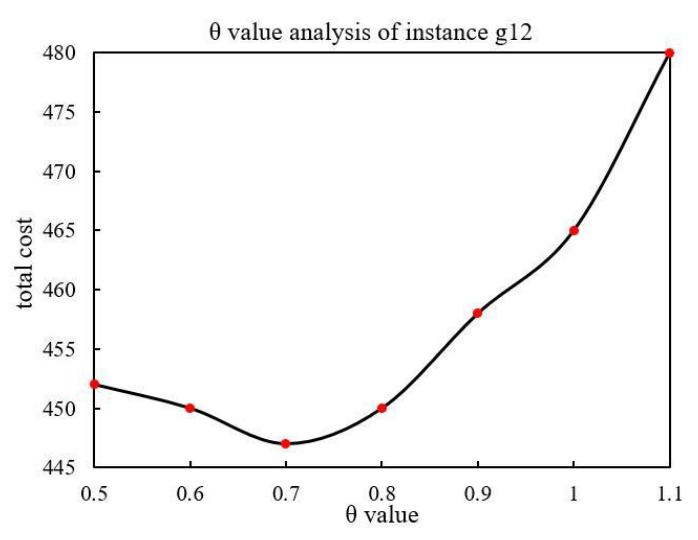

Figure 2. The total cost for different thresholds value $\theta$.

\subsection{Comparison between D-MAENS and IMA}

The two performances metrics evaluate the performance of the two algorithms. one is the inverted generational distance from reference set (IGD) [17], which indicates the convergence and diversity of a solution set in multi-objective problem. Another one is hypervolume (HV) [18], which mainly describes the convergence and diversity of a solution set in a sense. Each algorithm was run 30 times on each test instance and the average of the indicators was calculated for comparison. If an indicator of the algorithm is better than other algorithms, it will be bolded.

Table 2. Results of D-MAENS and IMA.

\begin{tabular}{|c|c|c|c|c|c|c|c|}
\hline \multirow{2}{*}{ Instances } & \multirow{2}{*}{$V$} & \multirow{2}{*}{$E$} & \multirow{2}{*}{$d$} & \multicolumn{2}{|c|}{ D-MAENS } & \multicolumn{2}{|c|}{ IMA } \\
\cline { 5 - 8 } & & & & IGD & HV & IGD & HV \\
\hline g1 & 12 & 22 & 1,12 & 8.64 & 658 & $\mathbf{8 . 2 8}$ & $\mathbf{7 3 5}$ \\
\hline g2 & 12 & 26 & 1,12 & 10.75 & $\mathbf{6 3 3}$ & $\mathbf{9 . 7 1}$ & 577 \\
\hline g3 & 12 & 22 & 1,12 & 9.86 & $\mathbf{9 0 0}$ & $\mathbf{8 . 7 9}$ & 719 \\
\hline g4 & 11 & 19 & 1,11 & $\mathbf{3 5 . 0 6}$ & $\mathbf{4 3 4}$ & 35.31 & 414 \\
\hline g5 & 13 & 26 & 1,13 & 15.42 & 851 & $\mathbf{1 3 . 0 9}$ & $\mathbf{1 0 5 6}$ \\
\hline g6 & 12 & 22 & 1,12 & 17.46 & $\mathbf{1 1 2 1}$ & $\mathbf{1 5 . 2 5}$ & 962 \\
\hline g7 & 12 & 22 & 1,12 & 18.43 & 1862 & $\mathbf{1 8 . 3 1}$ & $\mathbf{1 9 7 7}$ \\
\hline g8 & 27 & 46 & 1,27 & 23.36 & 291 & $\mathbf{2 3 . 1 1}$ & $\mathbf{3 8 0}$ \\
\hline g9 & 27 & 51 & 1,27 & $\mathbf{5 . 7 6}$ & 356 & 5.91 & $\mathbf{3 8 4}$ \\
\hline g10 & 12 & 25 & 1,12 & 16.17 & $\mathbf{1 6 2 0}$ & $\mathbf{1 6 . 0 8}$ & 1502 \\
\hline g11 & 22 & 45 & 1,22 & $\mathbf{1 3 . 5 5}$ & $\mathbf{5 1 2 9}$ & 14.60 & 1312 \\
\hline g12 & 13 & 23 & 1,13 & 7.27 & 900 & $\mathbf{7 . 1 5}$ & $\mathbf{9 7 0}$ \\
\hline g13 & 10 & 28 & 1,10 & $\mathbf{8 0 . 3 8}$ & 7624 & 93.98 & $\mathbf{9 5 0 0}$ \\
\hline g14 & 7 & 21 & 1,7 & $\mathbf{6 . 3 4}$ & 274 & 6.56 & $\mathbf{3 6 5}$ \\
\hline g15 & 7 & 21 & 1,7 & 1.37 & $\mathbf{4 1 2}$ & $\mathbf{1 . 2 2}$ & 150 \\
\hline g16 & 8 & 28 & 1,8 & 7.07 & 221 & $\mathbf{6 . 5 5}$ & $\mathbf{2 5 3}$ \\
\hline g17 & 8 & 28 & 1,8 & $\mathbf{1 . 1 5}$ & 76 & 1.68 & $\mathbf{7 8}$ \\
\hline g18 & 9 & 36 & 1,9 & 14.64 & $\mathbf{5 6 9}$ & $\mathbf{1 3 . 1 1}$ & 315 \\
\hline g19 & 8 & 11 & 1,8 & $\mathbf{0 . 0 2}$ & 55 & 0.023 & 55 \\
\hline g20 & 11 & 22 & 1,11 & 13.03 & 96 & $\mathbf{1 . 5 6}$ & $\mathbf{3 4 3}$ \\
\hline g21 & 11 & 33 & 1,11 & 14.97 & $\mathbf{4 9 5}$ & $\mathbf{9 . 2 3}$ & 352 \\
\hline g22 & 11 & 44 & 1,11 & 13.29 & 514 & $\mathbf{1 2 . 3 4}$ & $\mathbf{6 8 3}$ \\
\hline g23 & 11 & 55 & 1,11 & $\mathbf{1 0 . 1 3}$ & 363 & 10.64 & $\mathbf{4 3 2}$ \\
\hline & & & & & & & \\
\hline
\end{tabular}

Table 2 shows the attributes of the instances and the experimental result for the IMA performance. It can be seen that the IMA performs better than D-MAENS on 15 out of the 23 instances in the metric IGD. The average $\mathrm{HV}$ result is obtained by IMA and D-MAENS. It can be found that IMA obtains a better solution on 13 out of the 23 instances.
Table 3. The best solution of D-MAENS and IMA.

\begin{tabular}{|c|c|c|c|c|c|c|c|}
\hline \multirow{2}{*}{ Instances } & \multirow{2}{*}{$V$} & \multirow{2}{*}{$E$} & \multirow{2}{*}{$d$} & \multicolumn{2}{|c|}{ D-MAENS } & \multicolumn{2}{|c|}{ IMA } \\
\cline { 5 - 8 } & & & & $f_{1}$ & $f_{2}$ & $f_{1}$ & $f_{2}$ \\
\hline g1 & 12 & 22 & 1,12 & 320 & 76 & $\mathbf{3 0 0}$ & $\mathbf{6 3}$ \\
\hline g2 & 12 & 26 & 1,12 & 352 & 62 & $\mathbf{3 2 1}$ & $\mathbf{5 9}$ \\
\hline g3 & 12 & 22 & 1,12 & 307 & 63 & $\mathbf{2 6 3}$ & $\mathbf{5 9}$ \\
\hline g4 & 11 & 19 & 1,11 & 310 & 63 & $\mathbf{2 6 6}$ & $\mathbf{5 9}$ \\
\hline g5 & 13 & 26 & 1,13 & 379 & 63 & 361 & 64 \\
\hline g6 & 12 & 22 & 1,12 & 323 & 68 & $\mathbf{3 0 1}$ & $\mathbf{6 0}$ \\
\hline g7 & 12 & 22 & 1,12 & 345 & 72 & $\mathbf{3 2 6}$ & $\mathbf{7 2}$ \\
\hline g8 & 27 & 46 & 1,27 & 350 & 38 & 334 & 39 \\
\hline g9 & 27 & 51 & 1,27 & 305 & 37 & $\mathbf{2 8 6}$ & $\mathbf{3 5}$ \\
\hline g10 & 12 & 25 & 1,12 & 284 & 66 & $\mathbf{2 8 2}$ & $\mathbf{6 3}$ \\
\hline g11 & 22 & 45 & 1,22 & 397 & 84 & $\mathbf{3 8 7}$ & $\mathbf{8 4}$ \\
\hline g12 & 13 & 23 & 1,13 & 480 & 77 & 446 & 81 \\
\hline g13 & 10 & 28 & 1,10 & 536 & 151 & $\mathbf{5 1 5}$ & $\mathbf{1 2 0}$ \\
\hline g14 & 7 & 21 & 1,7 & 107 & 21 & $\mathbf{9 2}$ & $\mathbf{2 1}$ \\
\hline g15 & 7 & 21 & 1,7 & 58 & 15 & 58 & 15 \\
\hline g16 & 8 & 28 & 1,8 & 125 & 29 & $\mathbf{1 2 5}$ & $\mathbf{2 0}$ \\
\hline g17 & 8 & 28 & 1,8 & 91 & 13 & 91 & 13 \\
\hline g18 & 9 & 36 & 1,9 & 168 & 26 & $\mathbf{1 4 0}$ & $\mathbf{2 4}$ \\
\hline g19 & 8 & 11 & 1,8 & 55 & 21 & 55 & 21 \\
\hline g20 & 11 & 22 & 1,11 & $\mathbf{1 2 1}$ & $\mathbf{3 0}$ & 121 & 34 \\
\hline g21 & 11 & 33 & 1,11 & 158 & 30 & $\mathbf{1 2 8}$ & $\mathbf{2 8}$ \\
\hline g22 & 11 & 44 & 1,11 & 201 & 29 & 184 & 31 \\
\hline g23 & 11 & 55 & 1,11 & 233 & 26 & 223 & 28 \\
\hline
\end{tabular}

In the Table 3 , the nondominated solutions of the multi-objective functions are indicated. It can be seen that the IMA gains the better solutions for 14 out of 23 instances, while the D-MAENS only gains one better solutions. Figure 3 shows the nondominated solutions of the two algorithms after 30 independent runs. Due to limited space, the test instances of g1, g10, g14 and g21 only are displayed. It can be seen clearly that the IMA solution sets are better close to the Pareto-Optimal Front than D-MAENS.

Therefore, it can conclude that the performance of IMA is much better than the D-MAENS in the convergence and diversity of a solution set for MDMOCARP. The IMA considers the critical edge assignment problem in arc path optimization. The overall optimization of the arc path can effectively reduce the total cost and the largest consumption of vehicles.

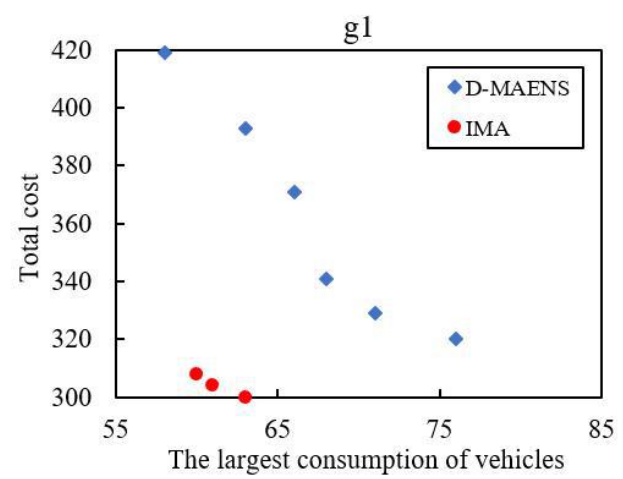



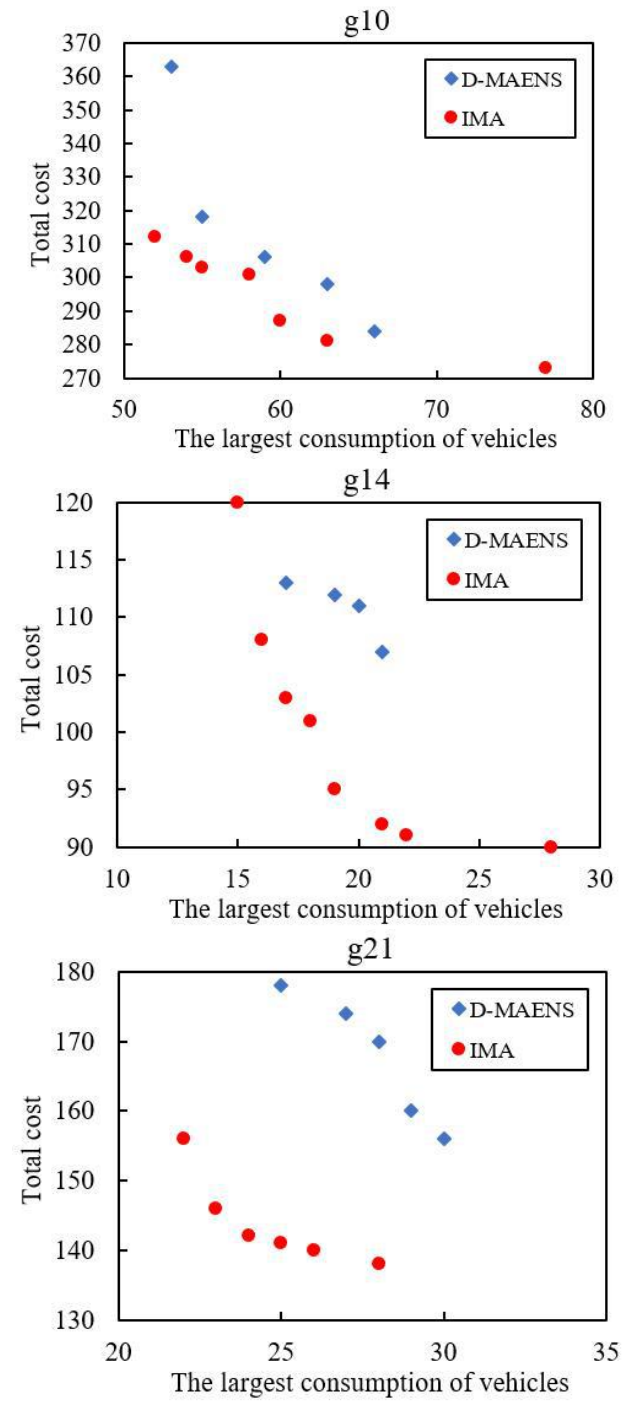

Figure 3. The nondominated solutions of the two algorithms.

\section{Conclusion}

In this paper, we study the multi-depot multi-objective Capacitated Arc Routing Problem, which is a more practical version of the CARP, and propose the multi-depot multi-objective CARP model. this model has two objective functions in the multi-depot, minimizing the total cost and minimize the largest consumption of vehicle. The IMA is proposed to solve the problem, which has the critical edge decision mechanism, the critical edge random allocation mechanism and a novel adaptive probability of local search with fitness of the individual.

Simulation results show that the IMA is competitive with respect to improving the quality of the non-dominant solution. Appropriate selection and distribution of critical edges can better optimize the routing, and reduce costs greatly.

In the future, some practical problems remain unsolved. Firstly, we will study the arc routing problem with the fuel consumption or the carbon dioxide emissions. Secondly, more efficient local searches probability needs to be studied to reduce computational costs and time. Finally, we only analyse the threshold, so we need to analyse more parameters in the future so that the algorithm achieves better results.

\section{Acknowledgment}

This paper is supported by No.18BGL012 from the China Social Science Foundation Project, No.2019031201028 from the China Hebei Social Science Development Research Project and No.2017GJJG021 from the China Hebei Higher Education Teaching Reform Research and Practice Project.

\section{References}

1. B. Golden, R. Wong. CARP, Net., 11, 305-315 (1981)

2. M. Tagmouti, M. Gendreau, J. Y. Potvin. DCARP., TRC., 19, 20-28(2009)

3. S. Nesmachnow, D. G. Rossit, J. Toutouh. Compari., ENDM., 69, 93-100(2018)

4. T. P. B. Vecchi, D. F. Surco, A. A. Constantino, et al. A seq., PSEP., 102, 238-250(2016)

5. R. D. Gabriel, V. Daniele, T. Fernando, et al. Visual., COR., 103, 13-34(2019)

6. R. Shang, B. Du, H. Ma. Immune., ASOC., 49, 748-758(2016)

7. D. Krushinsky, T. V. Woensel. An app., EJOR., 244, 100-109(2015)

8. E. B. Tirkolaee, I. Mahdavi, M. M. S. Esfahani. A robust., WasMan., 76, 138-146(2018)

9. A. Amberg, W. Domschke, S. Voß. Multi., EJOR., 124, 360-376(2000)

10. A. Kansou, A. Yassine. A two., ICCIE. IEEE, (2009).

11. L. Xing, P. Rohlfshagen, Y. Chen, et al. An evo., TEVC., 14, 356-374(2010)

12. F. B. D. Oliveira, R. Enayatifar, H. J. Sadaei, et al. A coo., ESWA., 43, 117-130(2016).

13. P. Lacomme, C. Prins, W. R. Cherif. Com., ANOR., 131, 159-185(2004)

14. Y. Mei, K. Tang, X. Yao. D-MAENS., TEVC., 15, 151-165(2011)

15. R. Shang, J. Wang, L. Jiao, et al. An impro., ASOC., 19, 343-361(2014)

16. R. Shang, K. Dai, L. Jiao, et al. Impro., TCYB., 46, 1000-1013(2016)

17. Q. Zhang, H. Li. MOEA/D., TEVC., 11, 712-731(2008)

18. T. Liu, Z. Jiang, N. Geng. A gen., FSM., 26, 540-564(2014) 